\title{
Constructing Spin-Adiabatic States for the Modeling of Spin-Crossing Reactions. I. A Shared-Orbital Implementation
}

\author{
Yunwen Tao, ${ }^{1}$ Zheng Pei, ${ }^{2}$ Nicole Bellonzi, ${ }^{3}$ Yuezhi Mao, ${ }^{4}$ a) Zhu Zou, ${ }^{1}$ Wanzhen Liang, ${ }^{2}$ Zhibo Yang, ${ }^{1}$ and \\ Yihan Shao ${ }^{1, b)}$ \\ ${ }^{1)}$ Department of Chemistry and Biochemistry, University of Oklahoma, Norman, OK 73019 \\ ${ }^{2)}$ State Key Laboratory of Physical Chemistry of Solid Surfaces, Collaborative Innovation Center of Chemistry for Energy Materials, \\ Fujian Provincial Key Laboratory of Theoretical and Computational Chemistry, and Department of Chemistry, \\ College of Chemistry and Chemical Engineering, Xiamen University, Xiamen 361005, \\ P. R. China \\ 3) Department of Chemistry, University of Pennsylvania, Philadelpha, PA 19104 \\ 4) Department of Chemistry, Stanford University, Stanford, CA 94305
}

(Dated: July 16, 2019)

In the modeling of spin-crossing reactions, it has become popular to directly explore the spin-adiabatic surfaces. Specifically, through constructing spin-adiabatic states from a two-state Hamiltonian (with spin-orbit coupling matrix elements) at each geometry, one can readily employ advanced geometry optimization algorithms to acquire a "transition state" structure, where the spin crossing occurs. In this work, we report the implementation of a fully-variational spin-adiabatic approach based on Kohn-Sham density functional theory spin states (sharing the same set of molecular orbitals) and the Breit-Pauli one-electron spin-orbit operator. For three model spin-crossing reactions [predissociation of $\mathrm{N}_{2} \mathrm{O}$, singlet-triplet conversion in $\mathrm{CH}_{2}$, and $\mathrm{CO}$ addition to $\mathrm{Fe}(\mathrm{CO})_{4}$ ], the spin-crossing points were obtained. Our results also indicated the Breit-Pauli one-electron spin-orbit coupling can vary significantly along the reaction pathway on the spin-adiabatic energy surface. On the other hand, due to the restriction that low-spin and high-spin states share the same set of molecular orbitals, the acquired spin-adiabatic energy surface shows a cusp (i.e. a first-order discontinuity) at the crossing point, which prevents the use of standard geometry optimization algorithms to pinpoint the crossing point. An extension with this restriction removed is being developed to achieve the smoothness of spin-adiabatic surfaces.

\section{INTRODUCTION}

In most chemical reactions, the total electron spin is conserved. However, if a reaction involves high-spin species, such as oxygen $\left({ }^{3} \mathrm{P}\right)$ and nitrogen $\left({ }^{4} \mathrm{~N}\right)$ atoms, molecular oxygen $\left({ }^{3} \mathrm{O}_{2}\right)$, and transition metals, ${ }^{1-8}$ a change in the electron spin might occur during the transition from the reactant to the product. Such spin-crossing reactions are known to be enabled by the spin-orbit coupling (SOC) between different spin states. ${ }^{9,10}$ While usually slower than their spin-conserved counterparts (especially in cases with a weak spin-orbit coupling), spin-crossing reactions can play an important role in biochemical processes, homogeneous/heterogeneous catalysis, energy storage/conversion, etc.

In the study of spin-crossing reactions, one can explore either the spin-diabatic or spin-adiabatic potential energy surfaces. In the spin-diabatic approach, the central task is to reach the crossing seam of two spin-diabatic potential energy surfaces, and then locate the minimum energy crossing point (MECP) on the crossing seam. ${ }^{1-14}$ This is illustrated in Figure 1a for a triplet-to-singlet reaction. Once the MECP is found, one can proceed to compute the spin-orbit coupling between the two spin states. The rate of a spin-crossing reaction is then assessed from the energy required to reach the MECP (i.e. activation energy) as well as the spin-orbit coupling value at the MECP (which controls the probability to

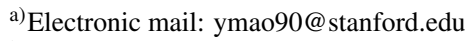

b)Electronic mail: yihan.shao@ou.edu
}

cross onto another spin-diabatic state). ${ }^{4}$ Over the years, the MECP approach has been successfully applied to numerous spin-crossing reactions. $1,3,4,7,15$

The spin-adiabatic approach, ${ }^{3,17}$ on the other hand, has gained a lot of momentum in last couple of years. ${ }^{16,18-22}$ As illustrated in Figure 1b, within this approach, one computes spin-orbit coupling at each geometry to construct the lowestenergy spin-adiabatic state, and then search for a transition state (TS) and the minimum energy pathway on the spinadiabatic surface. Such a so-called TS-SOC approach has been applied to a number of systems: $\mathrm{O}_{2}$ addition to gold hydride complexes and predissociation of $\mathrm{N}_{2} \mathrm{O}$ and $\mathrm{N}_{2} \mathrm{Se},{ }^{16,18,19}$ $\mathrm{CO}$ addition to $\mathrm{Fe}(\mathrm{CO})_{4}$ and hydrogen elimination from a tungsten complex, ${ }^{20} \mathrm{FeO}^{+}$reacting with $\mathrm{H}_{2}$ or $\mathrm{CH}_{4}$ and $\mathrm{Mn}^{+}$ reacting with $\mathrm{OCS}^{21}$ and several first-row transition metal cations $\left(\mathrm{Sc}^{+}\right.$to $\left.\mathrm{Cu}^{+}\right)$reacting with OCS molecule. ${ }^{22}$

For spin-crossing reactions with weak spin-orbit couplings between two spin states, MECP and TS-SOC approaches would yield very similar reaction mechanisms and rates, because the spin-orbit coupling causes only little structural difference between the MECP at the intersection of two spindiabatic surfaces and the TS on the spin-adiabatic surface. However, the TS-SOC approach has a clear computational advantage. Instead of exploring a high-dimensional crossing seam between two potential energy surfaces using an MECP algorithm, a TS-SOC calculation only requires the location of a first-order saddle point on a single potential energy surface. Thus, once the corresponding analytical nuclear gradient is available, a TS-SOC calculation can be readily handled using advanced TS search algorithms (such as partitioned rotational-function optimization, ${ }^{23}$ quadratic syn- 


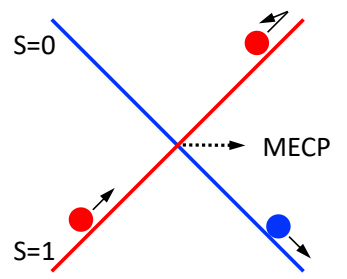

(a)

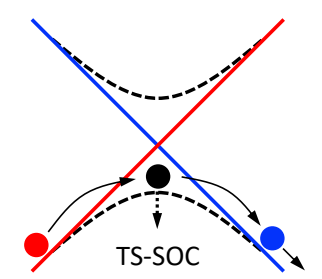

(b)

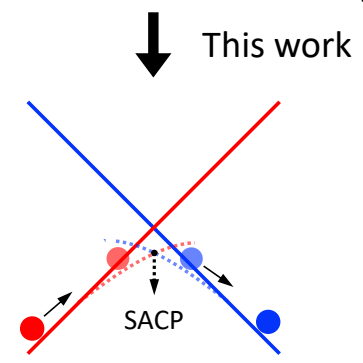

(c)

Figure 1. Schematic representation of three approaches for modeling spin-crossing reactions. Two existing approaches: ${ }^{16}$ (a) optimization of MECP between spin-diabatic surfaces; (b) optimization of transition state (TS) on the spin-adiabatic surface. This work: (c) optimization of spin-adiabatic cusp point (SACP) on the spin-adiabatic surface.

chronous transition method, ${ }^{24}$ dimer method, ${ }^{25-27}$ growing string method, ${ }^{28-30}$ and freezing string method ${ }^{31,32}$ ) that have been implemented in various quantum chemistry software packages.

In TS-SOC calculations, the spin-orbit coupling between spin-diabatic states has so far been formulated within the zeroorder regular approximation (ZORA). ${ }^{33,34}$ In the first TS-SOC implementation by Harvey and coworkers, ${ }^{16}$ ZORA coupling was computed on the fly (i.e. for each geometry) during the TS search. Later on, it was approximated to have a constant value in the two-state spin-mixing model (TSSMM) from Yang, Gagliardi, and $\operatorname{Truhlar}^{20}$ and adopted by Takayanagi and Nakatomi. ${ }^{21}$ Such an approximation removes the orbitaldependence of spin-orbit coupling values and thus greatly simplifies the evaluation of analytical nuclear gradients and Hessian matrices.

Inspired by the TS-SOC and TSSMM approaches, in this work, we explored the construction of spin-adiabatic surfaces using a different spin-orbit coupling Hamiltonian. Specifically, we employed the Breit-Pauli one-electron spin-orbit operator, ${ }^{9,35}$ which is also widely available for non-relativistic quantum chemistry calculations. ${ }^{36-40}$ Recently, the BreitPauli one-electron spin-orbit operator was also employed to construct spin-adiabatic excited states within both configuration interaction singles (CIS) ${ }^{41}$ and the Tamm-Dancoff approximation to time-dependent density functional theory (TDDFT/TDA $)^{42}$ frameworks. Similarly, we will adopt the original Breit-Pauli one-electron spin-orbit operator, with the expectation that the use of effective nuclear charges ${ }^{37}$ or a mean-field approach ${ }^{43}$ would reduce the spin-orbital coupling but retain a very similar picture for the reaction energetics.
In the construction of spin-adiabatic states, the component spin diabatic states can share the same set of molecular orbitals or have separately-optimized (i.e. unshared) molecular orbitals. An unshared-orbitals implementation based on Breit-Pauli spin-orbit coupling would require the solution of $z$-vector equations in the analytical gradient evaluation. Such an unshared-orbitals implementation will be reported in a subsequent publication.

In this work, we will explore a spin-restricted and sharedorbital implementation. Essentially, the same set of molecular orbitals will be adopted for the alpha and beta electrons, and for the two spin-diabatic states (i.e. restricted closed-shell singlet and restricted open-shell triplet) and thus resultant spinadiabatic states. Upon self-consistent-field (SCF) convergence, the energy of a shared-orbital spin-adiabatic groundstate is fully variational with respect to orbital rotations, thus allowing us to avoid the additional cost of solving $z$-vector equations during the stage of analytical gradient evaluation.

This article is organized as follows. Our new restricted-andshared-orbital method for acquring spin-adiabatic states will be introduced in Section II, with three model spin-crossing reactions and other computational details described in Section III. Computational results will be presented in Section IV. A discussion will be provided in Section V, mostly concerning an unintended consequence of orbital sharing between different spin states — our spin-adiabatic potential surface displays a cusp around the crossing point (as shown in Figure 1c). Because of this, an MECP-type algorithm was employed (instead of TS search algorithms) to reach the crossing point in our calculations. Finally, conclusions will be drawn in Section VI.

\section{METHODOLOGY}

\section{A. Spin-Adiabtic States}

In this work, we focus on molecular systems with an even number of electrons. The spin-adiabatic state of such systems will be described as a linear combination of two spin-diabatic Kohn-Sham states,

$$
\Psi^{\text {adia }}=C_{\text {sing }} \Psi^{\text {sing }}+C_{\text {trip }} \Psi^{\text {trip }}
$$

which are shown schematically in Figure 2. The restricted closed-shell singlet $(\mathrm{S}=0)$ and restricted open-shell triplet $(\mathrm{S}=1)$ states share the same core (doubly-occupied) orbitals to be labelled as $c, c^{\prime}$, and virtual orbitals labelled as $v$ and $v^{\prime}$. The difference between two spin-diabatic states thus occurs with open-shell orbitals labelled as $a, b$ : the first orbital $a$ is doubly occupied within the closed-shell singlet state; in contrast, $a$ and $b$ orbitals are both occupied by an alpha electron within the restricted open-shell triplet state.

The singlet and triplet states are coupled through SOC with the Hamiltonian being

$$
\mathbf{H}=\left(\begin{array}{ll}
E^{\text {sing }} & V^{\text {SOC }} \\
V^{\text {SOC }} & E^{\text {trip }}
\end{array}\right)
$$

where $E^{\text {sing }}$ and $E^{\text {trip }}$ are energies of two spin-diabatic states, 


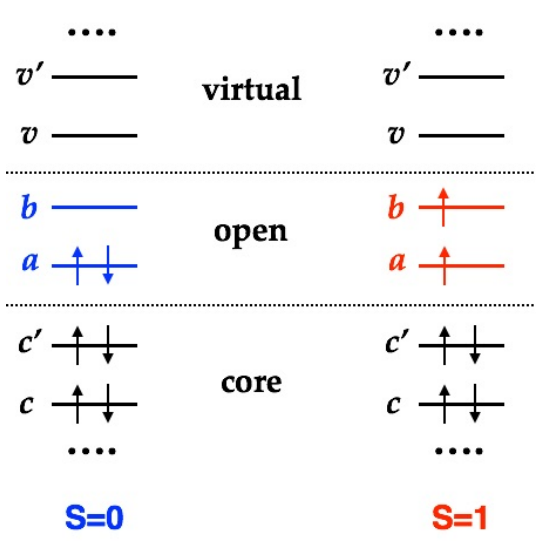

Figure 2. Restricted closed-shell singlet and open-shell triplet configurations for constructing spin-adiatic state.

and $V^{\mathrm{SOC}}$ is the spin-orbit coupling between these two states (to be defined below in Section II B). Solving this two-state problem, one arrives at two spin-adiabatic states in Eq.1 (with different mixing coefficients). The energy of the target (i.e. lower-energy) spin-adiabatic state is

$$
E^{\text {adia }}=\frac{E^{\text {sing }}+E^{\text {trip }}}{2}-\left[\frac{1}{4}\left(E^{\text {sing }}-E^{\text {trip }}\right)^{2}+\left(V^{\text {SOC }}\right)^{2}\right]^{\frac{1}{2}}
$$

To the extent that the spin-adiabatic state is a mixture of two "wavefunctions" built from a single set of restricted molecular orbitals, our method closely resembles the restricted openshell Kohn-Sham (ROKS) approach from van Voorhis and others for describing the lowest open-shell singlet state. ${ }^{44-46}$ As a result, our SCF procedure to converge molecular orbitals in Section II C will also be similar to the one for converging ROKS calculations.

\section{B. Spin-Orbit Coupling}

For molecules containing only main-group and first-row transition metal elements, which are the focus of this work, one can use the Breit-Pauli one-electron spin-orbit Hamiltonian

$$
\hat{V}^{\mathrm{SO}}=-\frac{\alpha_{0}^{2}}{2} \sum_{j, A} \frac{Z_{A}}{\left|\vec{r}_{j A}\right|^{3}}\left(\vec{r}_{j A} \times \hat{p}_{j}\right) \cdot \hat{s}_{j}
$$

where $\alpha_{0}$ is the fine structure constant $(\sim 1 / 137), Z_{A}$ is the nuclear charge of the $A$-th atom, $\vec{r}_{j A}$ is the vector from the $A$ th atom to the $j$-th electron, $\hat{p}_{j}\left(\hat{s}_{j}\right)$ is the momentum (spin) operator for the $j$-th electron. The three components of the Breit-Pauli operator

$$
\hat{V}^{\mathrm{SO}}=\left\{\hat{V}_{x}^{\mathrm{SO}}, \hat{V}_{y}^{\mathrm{SO}}, \hat{V}_{z}^{\mathrm{SO}}\right\}
$$

can be represented in an atomic-orbital basis $\left(\phi_{\mu}, \phi_{v}\right)$

$$
V_{m, \mu v}^{\mathrm{SO}}=\left\langle\phi_{\mu}\left|\hat{V}_{m}^{\mathrm{SO}}\right| \phi_{v}\right\rangle, \quad m=\mathrm{x}, \mathrm{y}, \mathrm{z}
$$

Using these three matrices, one can compute the SOC between two open-shell orbitals (with orbital coefficients being $C_{\mu a}$ and $\left.C_{v b}\right)$

$$
V_{m, a b}^{\mathrm{SO}}=\sum_{\mu v} C_{\mu a} V_{m, \mu v}^{\mathrm{SO}} C_{v b}, \quad m=\mathrm{x}, \mathrm{y}, \mathrm{z}
$$

and express the SOC element in Eq. 2 as

$$
V^{\mathrm{SOC}}=\left(\sum_{m=x, y, z}\left|V_{m, a b}^{\mathrm{SO}}\right|^{2}\right)^{\frac{1}{2}}
$$

which is the total spin-orbit coupling between the singlet and all three triplet components $\left(m_{s}=1,0,-1\right)$.

\section{Self Consistent Field Procedure}

Since the two spin-diabatic states share the same set of molecular orbitals, these orbitals can be variationally optimized to minimize the spin-adiabtic state energy in Eq.3. During the SCF cycles, the orbitals will be updated using the following rotations

$$
\Theta=\left(\begin{array}{cccc}
\mathbf{0} & -\Theta_{\mathrm{ac}}^{\dagger} & -\Theta_{\mathrm{bc}}^{\dagger} & -\Theta_{\mathrm{vc}}^{\dagger} \\
\Theta_{\mathrm{ac}} & \mathbf{0} & -\Theta_{\mathrm{ba}}^{\dagger} & -\Theta_{\mathrm{va}}^{\dagger} \\
\Theta_{\mathrm{bc}} & \Theta_{\mathrm{ba}} & \mathbf{0} & -\Theta_{\mathrm{vb}}^{\dagger} \\
\Theta_{\mathrm{vc}} & \Theta_{\mathrm{va}} & \Theta_{\mathrm{vb}} & \mathbf{0}
\end{array}\right)
$$

The derivative of the energy in Eq.3 with respect to these orbital rotations yields the Fock matrix,

$$
\mathbf{F}^{\mathrm{adia}}=\left(\begin{array}{llll}
\mathbf{F}_{\mathrm{cc}} & \mathbf{F}_{\mathrm{ca}} & \mathbf{F}_{\mathrm{cb}} & \mathbf{F}_{\mathrm{cv}} \\
\mathbf{F}_{\mathrm{ac}} & \mathbf{F}_{\mathrm{aa}} & \mathbf{F}_{\mathrm{ab}} & \mathbf{F}_{\mathrm{av}} \\
\mathbf{F}_{\mathrm{bc}} & \mathbf{F}_{\mathrm{ba}} & \mathbf{F}_{\mathrm{bb}} & \mathbf{F}_{\mathrm{bv}} \\
\mathbf{F}_{\mathrm{vc}} & \mathbf{F}_{\mathrm{va}} & \mathbf{F}_{\mathrm{vb}} & \mathbf{F}_{\mathrm{vv}}
\end{array}\right) .
$$

It contains three terms

$$
\mathbf{F}^{\text {adia }}=\frac{\partial E^{\text {adia }}}{\partial E^{\text {sing }}} \mathbf{F}^{\text {sing }}+\frac{\partial E^{\text {adia }}}{\partial E^{\text {trip }}} \mathbf{F}^{\text {trip }}+\frac{\partial E^{\text {adia }}}{\partial V^{\text {SOC }}} \Delta \mathbf{F}^{\text {SOC }}
$$

The restricted closed-shell singlet Fock matrix is computed as usual

$$
\mathbf{F}^{\text {sing }}=\left(\begin{array}{llll}
\mathbf{F}_{\mathrm{cc}}^{\text {sing }} & \mathbf{F}_{\mathrm{ca}}^{\text {sing }} & \mathbf{F}_{\mathrm{cb}}^{\text {sing }} & \mathbf{F}_{\mathrm{cv}}^{\text {sing }} \\
\mathbf{F}_{\mathrm{ac}}^{\text {sing }} & \mathbf{F}_{\mathrm{aa}}^{\text {sing }} & \mathbf{F}_{\mathrm{ab}}^{\text {sing }} & \mathbf{F}_{\mathrm{av}}^{\text {sing }} \\
\mathbf{F}_{\mathrm{bc}}^{\text {sing }} & \mathbf{F}_{\mathrm{ba}}^{\text {sing }} & \mathbf{F}_{\mathrm{bb}}^{\text {sing }} & \mathbf{F}_{\mathrm{bv}}^{\text {sing }} \\
\mathbf{F}_{\mathrm{vc}}^{\text {sing }} & \mathbf{F}_{\mathrm{va}}^{\text {sing }} & \mathbf{F}_{\mathrm{vb}}^{\text {sing }} & \mathbf{F}_{\mathrm{vv}}^{\text {sing }}
\end{array}\right)
$$

The off-diagonal blocks ( $c a, c b, c v, a v, b v$ ) of the restricted open-shell triplet effective Fock matrix (with two unpaired alpha electrons) can be written as energy derivatives with respect to orbital rotations in Eq.9,

$$
\mathbf{F}=\frac{\partial E}{\partial \mathbf{P}^{\alpha}} \frac{\partial \mathbf{P}^{\alpha}}{\partial \Theta}+\frac{\partial E}{\partial \mathbf{P}^{\beta}} \frac{\partial \mathbf{P}^{\beta}}{\partial \Theta}=\mathbf{F}^{\alpha} \frac{\partial \mathbf{P}^{\alpha}}{\partial \Theta}+\mathbf{F}^{\beta} \frac{\partial \mathbf{P}^{\beta}}{\partial \Theta}
$$


where the $\mathbf{P}^{\alpha}$ and $\mathbf{P}^{\beta}$ are alpha and beta density matrices. The triplet effective Fock matrix can then be written as ${ }^{44}$

$$
\mathbf{F}^{\text {trip }}=\left(\begin{array}{cccc}
\mathbf{R}_{\mathrm{cc}} & \mathbf{F}_{\mathrm{ca}}^{\mathrm{trip}, \beta} & \mathbf{F}_{\mathrm{cb}}^{\mathrm{trip}, \beta} & \mathbf{F}_{\mathrm{cv}}^{\mathrm{trip}, \alpha}+\mathbf{F}_{\mathrm{cv}}^{\mathrm{trip}, \beta} \\
\mathbf{F}_{\mathrm{ac}}^{\mathrm{trip}, \beta} & \mathbf{R}_{\mathrm{aa}} & \mathbf{R}_{\mathrm{ab}} & \mathbf{F}_{\mathrm{av}}^{\mathrm{trip}, \alpha} \\
\mathbf{F}_{\mathrm{bc}}^{\mathrm{trip}, \beta} & \mathbf{R}_{\mathrm{ba}} & \mathbf{R}_{\mathrm{bb}} & \mathbf{F}_{\mathrm{bv}}^{\mathrm{trip}, \alpha} \\
\mathbf{F}_{\mathrm{vc}}^{\mathrm{trip}, \alpha}+\mathbf{F}_{\mathrm{vc}}^{\mathrm{trip}, \beta} & \mathbf{F}_{\mathrm{va}}^{\mathrm{trip}, \alpha} & \mathbf{F}_{\mathrm{vb}}^{\mathrm{trip}, \alpha} & \mathbf{R}_{\mathrm{vv}}
\end{array}\right)
$$

which reflects the fact that, in the $\mathrm{M}_{s}=1$ triplet, $c v$ rotations affect both alpha and beta density/Fock matrices, but $c a$ and $c b$ ( $a v$ and $b v)$ rotations only perturb beta (alpha) density matrices. The diagonal blocks $(\mathbf{R})$ are not uniquely defined, ${ }^{47,48}$ and in our implementation we shall adopt the Binkley-PopleDobosh operators, ${ }^{49}$

$$
\begin{aligned}
& \mathbf{R}_{\mathrm{cc}}=\frac{1}{2}\left(\mathbf{F}_{\mathrm{cc}}^{\mathrm{trip}, \alpha}+\mathbf{F}_{\mathrm{cc}}^{\mathrm{trip}, \beta}\right) \\
& \mathbf{R}_{\mathrm{aa}}=\mathbf{F}_{\mathrm{aa}}^{\mathrm{trip}, \alpha} \\
& \mathbf{R}_{\mathrm{ab}}=\mathbf{R}_{\mathrm{ba}}=\mathbf{F}_{\mathrm{ab}}^{\mathrm{trip}, \alpha} \\
& \mathbf{R}_{\mathrm{bb}}=\mathbf{F}_{\mathrm{bb}}^{\mathrm{trip}, \alpha} \\
& \mathbf{R}_{\mathrm{vv}}=\mathbf{F}_{\mathrm{vv}}^{\mathrm{trip}, \beta}
\end{aligned}
$$

The non-zero elements of $\Delta \mathbf{F}^{\mathrm{SOC}}$ are

$$
\begin{aligned}
& \Delta \mathbf{F}_{a c}^{\mathrm{SOC}}=\Delta \mathbf{F}_{c a}^{\mathrm{SOC}}=-\frac{2}{V^{\mathrm{SOC}}}\left(\vec{V}_{c b}^{\mathrm{SO}} \cdot \vec{V}_{a b}^{\mathrm{SO}}\right) \\
& \Delta \mathbf{F}_{b c}^{\mathrm{SOC}}=\Delta \mathbf{F}_{c b}^{\mathrm{SOC}}=-\frac{2}{V^{\mathrm{SOC}}}\left(\vec{V}_{a c}^{\mathrm{SO}} \cdot \vec{V}_{a b}^{\mathrm{SO}}\right) \\
& \Delta \mathbf{F}_{v a}^{\mathrm{SOC}}=\Delta \mathbf{F}_{a v}^{\mathrm{SOC}}=\frac{2}{V^{\mathrm{SOC}}}\left(\vec{V}_{v b}^{\mathrm{SO}} \cdot \vec{V}_{a b}^{\mathrm{SO}}\right) \\
& \Delta \mathbf{F}_{v b}^{\mathrm{SOC}}=\Delta \mathbf{F}_{b v}^{\mathrm{SOC}}=\frac{2}{V^{\mathrm{SOC}}}\left(\vec{V}_{a v}^{\mathrm{SO}} \cdot \vec{V}_{a b}^{\mathrm{SO}}\right)
\end{aligned}
$$

Putting the singlet, triplet, and SOC components together according to Eq.11, the total Fock matrix in Eq.10 is obtained. To ensure its off-diagonal blocks ( $c a, c b, c v, a v, b v$, and $a b$ ) vanish at SCF convergence, we defined a mixed density matrix,

$$
\mathbf{P}_{c}-\mathbf{P}_{a}+\mathbf{P}_{b}=\left(\begin{array}{cccc}
\mathbf{2} & \mathbf{0} & \mathbf{0} & \mathbf{0} \\
\mathbf{0} & -\mathbf{1} & \mathbf{0} & \mathbf{0} \\
\mathbf{0} & \mathbf{0} & \mathbf{1} & \mathbf{0} \\
\mathbf{0} & \mathbf{0} & \mathbf{0} & \mathbf{0}
\end{array}\right)
$$

where $\mathbf{P}_{c}$ refers to a diagonal matrix with a value of 2 for core orbitals only, $\mathbf{P}_{a}\left(\mathbf{P}_{b}\right)$ matrix with a non-zero value of 1 for the $(a, a)$-th $((b, b)$-th) element only. The SCF error vector (e) and error $(e)$ are then defined as

$$
\begin{aligned}
\mathbf{e} & =\left[\mathbf{F}^{\mathrm{adia}}, \mathbf{P}_{c}-\mathbf{P}_{a}+\mathbf{P}_{b}\right] \\
& =\left(\begin{array}{cccc}
\mathbf{0} & -3 \mathbf{F}_{\mathrm{ca}} & -\mathbf{F}_{\mathrm{cb}} & -2 \mathbf{F}_{\mathrm{cv}} \\
3 \mathbf{F}_{\mathrm{ac}} & \mathbf{0} & 2 \mathbf{F}_{\mathrm{ab}} & \mathbf{F}_{\mathrm{av}} \\
\mathbf{F}_{\mathrm{bc}} & -2 \mathbf{F}_{\mathrm{ba}} & \mathbf{0} & -\mathbf{F}_{\mathrm{bv}} \\
2 \mathbf{F}_{\mathrm{vc}} & -\mathbf{F}_{\mathrm{va}} & \mathbf{F}_{\mathrm{vb}} & 0
\end{array}\right) \\
e & =\|\mathbf{e}\|
\end{aligned}
$$

The minus sign in $\mathbf{P}_{c}-\mathbf{P}_{a}+\mathbf{P}_{b}$ was chosen so that the $(a, b)$ th element in the error vector (e) does not vanish until SCF convergence.

\section{Analytical Nuclear Gradient}

From Eq.3, we can derive its nuclear gradient,

$$
E^{\text {adia },[x]}=\frac{\partial E^{\text {adia }}}{\partial E^{\text {sing }}} E^{\text {sing, },[x]}+\frac{\partial E^{\text {adia }}}{\partial E^{\text {trip }}} E^{\text {trip },[x]}+\frac{\partial E^{\text {adia }}}{\partial V^{\text {SOC }}} V^{\text {SOC },[x]}
$$

where $[x]$ stands for the first derivative with respect to $x$. Here, the nuclear gradient of the spin-orbit coupling (Eq.8) is

$$
V^{\mathrm{SOC},[x]}=\frac{1}{V^{\mathrm{SOC}}} \sum_{m=x, y, z} V_{m, a b}^{\mathrm{so}} V_{m, a b}^{\mathrm{so},[x]}
$$

The derivative of the spin-orbit coupling between two singlyoccupied orbitals $(a, b)$ is

$$
\begin{aligned}
V_{m, a b}^{\mathrm{so},[x]} & =-\frac{1}{2}\left[\left(C C^{\dagger}\right) V_{m}^{\mathrm{SO}}\left(C_{b} C_{a}^{\dagger}\right)+\left(C_{b} C_{a}^{\dagger}\right) V_{m}^{\mathrm{SO}}\left(C C^{\dagger}\right)\right] \cdot \mathbf{S}^{[x]} \\
& +\left(C_{b} C_{a}^{\dagger}\right) \cdot \mathbf{V}_{m}^{\mathrm{SO},[\mathrm{x}]}
\end{aligned}
$$

where $\mathbf{S}^{[x]}$ and $\mathbf{V}_{m}^{\mathrm{SO},[\mathrm{x}]}$ are nuclear derivatives of the overlap and spin-orbit coupling matrices in the atomic basis.

\section{COMPUTATIONAL DETAILS}

The approach of constructing spin-adiabatic state outlined above was implemented in a development version of the QCHEM 5.1 software package. ${ }^{50}$ For systems with unbonded atoms $\left[\mathrm{N}_{2} \mathrm{O}\right.$ at long $\mathrm{N}-\mathrm{O}$ distances], a procedure combining direct inversion in iterative subspace (DIIS) ${ }^{51}$ and geometric direct mininzation (GDM) ${ }^{52}$ was used to achieve SCF convergence.

In this work, we study three spin-crossing reaction systems: $\mathrm{N}_{2} \mathrm{O}, \mathrm{CH}_{2}$ and $\mathrm{Fe}(\mathrm{CO})_{5}$. They were modeled with density functional theory (DFT) at the levels of B3LYP/6-31+G(d), ${ }^{53-56}$ B3LYP/6-311G(d,p), ${ }^{57}$ and M06L/def2-TZVPP, ${ }^{58-60}$ respectively. The DFT calculations were carried out on a pruned $(75,302)$ integration grid. ${ }^{61}$ We note that a highly accurate description for both $\mathrm{N}_{2} \mathrm{O}$ and $\mathrm{CH}_{2}$ systems would require CASSCF and other advanced electronic 
structure methods that properly account for the static correlation effect. The purpose of performing DFT calculations on these two systems in this work is mainly to explore the feasibility of computing their spin-adibatic states within DFT framework.

The study of $\mathrm{N}_{2} \mathrm{O}$ is rather straightforward, where onedimensional scan was performed while the molecule remains linear. For the $\mathrm{CH}_{2}$ and $\mathrm{Fe}(\mathrm{CO})_{5}$ systems, the analytical gradients formulated in Sec. II.D was used for optimizing the geometries. Specifically, a modified version of Harvey's MECP program ${ }^{14}$ called sobMECP by Lu (http://sobereva.com/286) was employed to locate the spin-adiabatic cusp point (SACP) on the spin-adiabatic potential energy surfaces. A detailed explanation for why our calculations lead to a cusp will be provided in Section V. At each geometry, the sobMECP program utilizes the energy and gradient of two mixed-spin SCF solutions, which were obtained using singlet and triplet SCF guess, respectively, and were thus dominated by either the $S_{0}$ or $\mathrm{T}_{1}$ state.

After SACP was located, the minimum energy path (MEP) for the $\mathrm{CH}_{2}$ system was generated by integrating along the nuclear gradients in Cartesian coordinates using an external script. For the $\mathrm{Fe}(\mathrm{CO})_{5}$ system, the pathway connecting reactant, SACP, and product was generated by a linear interpolation of Z-matrix. For a comparison, spin-orbit coupling matrix elements for the $\mathrm{Fe}(\mathrm{CO})_{5}$ system were also computed using the spin-orbit mean-field (SOMF) approach in the ORCA 4.1.1 package. $^{62}$

\section{RESULTS}

In this work, we have applied the restricted-and-sharedorbital formulation to model the aforementioned three spincrossing reactions as test cases.

\section{A. Predissociation of $\mathrm{N}_{2} \mathrm{O}$}

Nitrous oxide $\left(\mathrm{N}_{2} \mathrm{O}\right)$, also known as laughing gas has been widely used as an anaesthetic in surgery since $1844 .{ }^{63}$ Its mode-specific predissociation has been subjected to many theoretical studies, ${ }^{64-68}$ because it involves the crossing of two diabatic potential energy surfaces with different spins. ${ }^{69}$

In the dissociation pathway of oxygen atom from the $\mathrm{N}_{2} \mathrm{O}$ molecule, the entire reaction complex retains a linear geometry. Therefore, simultaneous linear interpolations were carried out for the N-N and N-O distances. For simplicity, only the $\mathrm{N}-\mathrm{O}$ distance will be shown as the reaction coordinate connecting the reactant (singlet $\mathrm{N}_{2} \mathrm{O}$ molecule) and the product $\left(\mathrm{N}_{2}+\mathrm{O}\right.$ complex, where oxygen atom adopts a triplet electronic configuration).

Figure 3 shows the spin-adiabatic potential energy surface along the reaction path as well as the spin-orbit coupling strength. Our restricted-and-shared-orbital calculations converged correctly to the first triplet state $\left({ }^{3} \Pi\right)$ when the $\mathrm{N}-\mathrm{O}$ bond is elongated. ${ }^{67}$ The highest energy point is found when the N-O distance is $1.7801 \AA$, which is in line with theoretical study by Hwang and Mebel. ${ }^{68}$ However, our calculated energy barrier of $70.6 \mathrm{kcal} / \mathrm{mol}$, which is similar to previous DFT results, ${ }^{68}$ is about $10 \mathrm{kcal} / \mathrm{mol}$ higher than experimental data ${ }^{70}$ and other theoretical investigations based on MRCI calculations. ${ }^{67,68}$ This is largely caused by the fact that conventional spin-restricted DFT calculation, which is based on single Slater determinant wavefunction, fails to capture the multi-reference character of this system upon the dissociation of the oxygen atom.

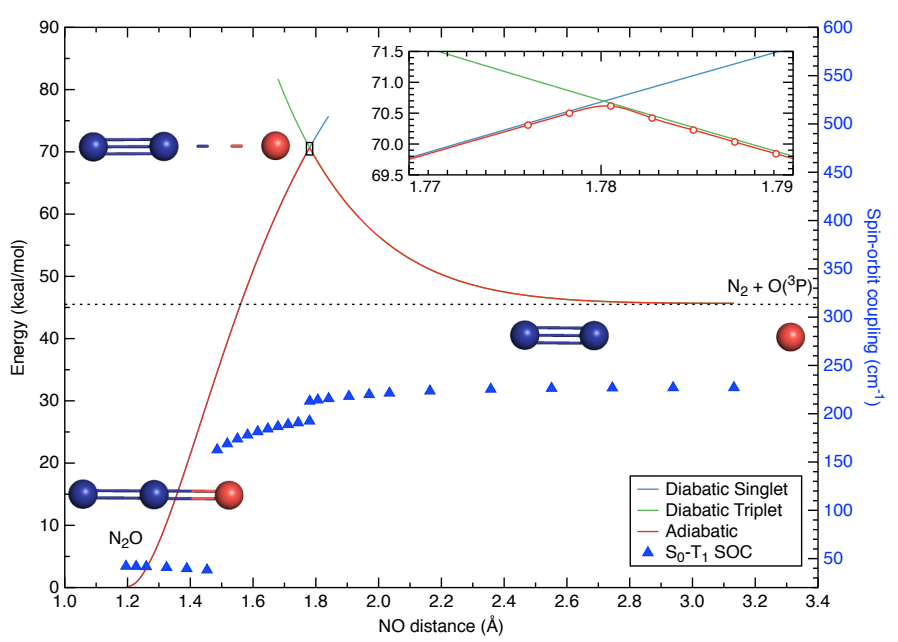

Figure 3. Energy profile and SOC strengths for $\mathrm{N}_{2} \mathrm{O}$ predissociation calculated at B3LYP/6-31+G(d) level. Blue and green curves represent energies for diabatic singlet and triplet states, respectively. Red curve stands for the spin-adiabatic state energy. Blue triangle dots show the change in the $\mathrm{S}_{0}-\mathrm{T}_{1}$ spin-orbit coupling strength along the reaction path.

At the SACP, the spin-orbit coupling strength is calculated to be around $200 \mathrm{~cm}^{-1}$, which is larger than $\sim 90 \mathrm{~cm}^{-1}$ calculated with full Breit-Pauli operator (including both oneelectron spin-orbit and two-electron spin-other-orbit contributions) and CASSCF. ${ }^{67,68}$ This happens because Eq.4 does not include the two-electron term in the Breit-Pauli spin-orbit Hamiltonian. Interestingly, the spin-orbit coupling strength, which is relatively low at short N-O bond lengths, has a sudden increase to $150 \mathrm{~cm}^{-1}$ when the $\mathrm{N}-\mathrm{O}$ bond length increases to $1.5 \AA$. This change in the $V^{\mathrm{SOC}}$ value was traced to a orbital swapping at that bond length - the second open-shell orbital $b$ changes from a $\pi$ orbital into a $\sigma$ orbital. Meanwhile, the first open-shell orbital $a$ stays the same as a $\pi$ orbital.

\section{B. ${ }^{1} A_{1} \rightarrow{ }^{3} B_{1}$ Conversion in $\mathrm{CH}_{2}$}

Carbene $\left(\mathrm{CH}_{2}\right)$ is one of the most widely studied intermediates in organic synthesis with divergent reactivity arising from its varioius electronic structure. ${ }^{71}$ In gas-phase, its most stable state is a triplet $\left({ }^{3} B_{1}\right)$. It can be excited into a singlet state $\left({ }^{1} A_{1}\right)$ with an adiabatic excitation energy of $9.0 \mathrm{kcal} / \mathrm{mol} .{ }^{72}$ The process for the excited carbene to return to its ground state is a simple spin-crossing reaction. 
Table I. Relative energies (kcal/mol), $\mathrm{CH}$ bond length $\left(R_{C H}, \AA\right)$ and $\mathrm{HCH}$ bond angle $\left(\alpha_{\mathrm{HCH}},{ }^{\circ}\right)$ for three key geometries on the spinadiabatic potential energy surface calculated at B3LYP/6-311G(d,p) level.

\begin{tabular}{lccc}
\hline \hline & ${ }^{1} A_{1}$ & $\mathrm{SACP}$ & ${ }^{3} B_{1}$ \\
\hline Energy & 0.00 & 0.00 & -11.40 \\
$R_{C H}$ & 1.1148 & 1.1146 & 1.0802 \\
$\alpha_{H C H}$ & 101.08 & 101.23 & 133.52 \\
\hline \hline
\end{tabular}

Through computing two mixed-spin solutions (dominated by ${ }^{3} B_{1}$ and ${ }^{1} A_{1}$ states, respectively) at each geometry, the local minima and SACP on the crossing seam can be identified as shown in Table I.

As shown in Figure 4 , the ${ }^{1} A_{1}$ minimum is quite close to SACP in terms of geometry as well as energy, the barrierless activation towards SACP leads to an efficient transition into the ${ }^{3} B_{1}$ state induced by spin-orbit coupling. Here a minimum energy path has been mapped out starting from SACP, the $\mathrm{HCH}$ angle increases gradually while the $\mathrm{CH}$ bond shortens drastically first followed by a slight elongation.

Our calculation shows the spin-orbit coupling along the reaction path between ${ }^{3} B_{1}$ and ${ }^{1} A_{1}$ electronic states is in the range of 43-59 $\mathrm{cm}^{-1}$, which is larger than the spin-orbit coupling values $\left(12-13 \mathrm{~cm}^{-1}\right)$ calculated by using full Breit-Pauli operator. ${ }^{73,74}$

\section{C. $\mathrm{CO}$ addition to $\mathrm{Fe}(\mathrm{CO})_{4}$}

The binding of carbon monoxide (CO) with iron tetracarbonyl $\left(\mathrm{Fe}(\mathrm{CO})_{4}\right)$ in its triplet ground state could lead to iron pentacarbonyl $\left(\mathrm{Fe}(\mathrm{CO})_{5}\right)$ in a closed-shell singlet state. This spin-crossing reaction, which was first discovered by Seder, Ouderkirk and Weitz, ${ }^{75}$ has been employed as an ideal but challenging test example for theoretical modeling by Harvey ${ }^{76}$ and by Yang, Gagliardi and Truhlar, ${ }^{20}$ due to its relatively small energy barrier above the triplet reactant.

As shown in Figure 5, our calculations provided a qualitatively reasonable energy barrier of $1.93 \mathrm{kcal} / \mathrm{mol}$ and the SACP geometry is more similar to the transition state structure identified in Yang/Gagliardi/Truhlar's work than the MECP structure with $C_{2 v}$ symmetry obtained by Harvey, ${ }^{20,76,77}$ although our calculated Fe-C distance is the shortest. As a result of spin-orbit coupling, the SACP is $0.03 \mathrm{kcal} / \mathrm{mol}$ lower in energy than the crossing point of two diabatic states.

Along the reaction path, the spin-orbit coupling strength is around $150-230 \mathrm{~cm}^{-1}$. However, near the product geometry, the spin-orbit coupling value increased to $285 \mathrm{~cm}^{-1}$, which is due to a change in the molecular symmetry from $C_{1}$ to $D_{3 h}$ and a related character change in the second openshell orbital $b$. For a comparison, we calculated the spinorbit coupling strength between $\mathrm{S}_{0}$ and $\mathrm{T}_{1}$ states using spinorbit mean-field (SOMF) Hamiltonian, which should have a higher accuracy. ${ }^{9,43}$ Smaller $\mathrm{S}_{0}-\mathrm{T}_{1}$ spin-orbit coupling around $90 \mathrm{~cm}^{-1}$ were produced in SOMF calculations, with the value

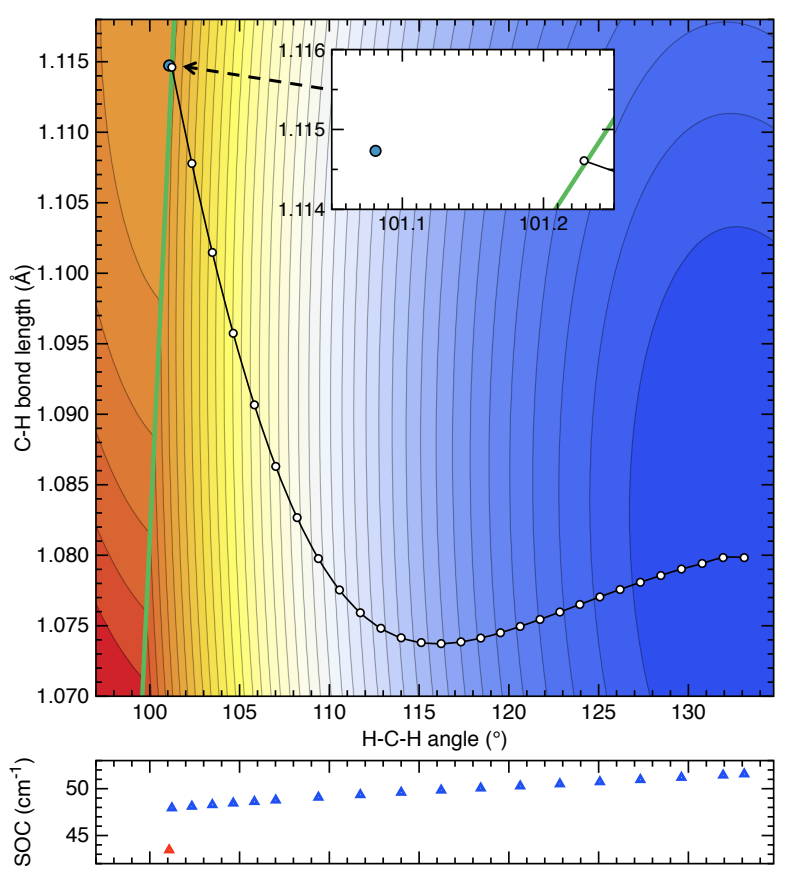

Figure 4. Minimum energy path for the singlet-triplet conversion of $\mathrm{CH}_{2}$ from the spin-adiabatic cusp point (SACP) towards the local minimum of the ${ }^{3} B_{1}$-dominated portion of spin-adiabatic potential energy surface. The underlying contour plot shows the energy landscape of the spin-adiabatic state with red color for higher energy and blue for lower energy. The green line is the intersection curve of two portions of the spin-adiabatic potential energy surface dominated by ${ }^{1} A_{1}$ and ${ }^{3} B_{1}$, respectively. The blue dot corresponds to the local minimum point on the ${ }^{1} A_{1}$-dominated portion of the spin-adiabatic PES. The white dot lying on the green line is the SACP, and the white dot at the rightmost is the local minimum point on the ${ }^{3} B_{1}$-dominated portion of the spin-adiabatic surface. Lower panel shows the spinorbit coupling strength $V^{\mathrm{SOC}}$ as a function of $\mathrm{HCH}$ angle. The red and blue triangle dots stand for the SOC matrix elements for ${ }^{1} A_{1}$ and ${ }^{3} B_{1}$-dominated spin-adiabatic states, respectively.

Table II. Geometric parameters (as defined in Figure 6) of the transition structure geometries for the $\mathrm{Fe}(\mathrm{CO})_{5}$ spin-crossing reaction complex. Bond lengths and angles are in Angstrom and degree, respectively. MECP: the MECP geometry reported in Harvey's work. ${ }^{76,77}$ TSSMM: the transition state geometry identified with TSSMM method by Truhlar and co-workers. ${ }^{20}$ SACP: the SACP geometry shown in Figure 5.

\begin{tabular}{lcrrrcc}
\hline \hline & $\alpha$ & $\beta$ & \multicolumn{1}{c}{$\gamma$} & $r$ & $r_{a x}$ & $r_{e q}$ \\
\hline MECP & 135 & 165.8 & 102.3 & 2.557 & 1.901 & 1.843 \\
TSSMM & 130.9 & 152.8 & 97.6 & 2.685 & 1.871 & 1.837 \\
SACP & 136.4 & 155.8 & 96.5 & 2.409 & 1.867 & 1.827 \\
\hline \hline
\end{tabular}

decreasing to nearly zero for the product geometry. The reason why SOMF gives much smaller $\mathrm{S}_{0}-\mathrm{T}_{1}$ spin-orbit couplings is in part because of the mean-field treatment and in part because the SOMF Hamiltonian is based on the TDDFT/TDA framework. In the $\mathrm{Fe}(\mathrm{CO})_{5}$ case, the $\mathrm{T}_{1}$ state clearly involves 


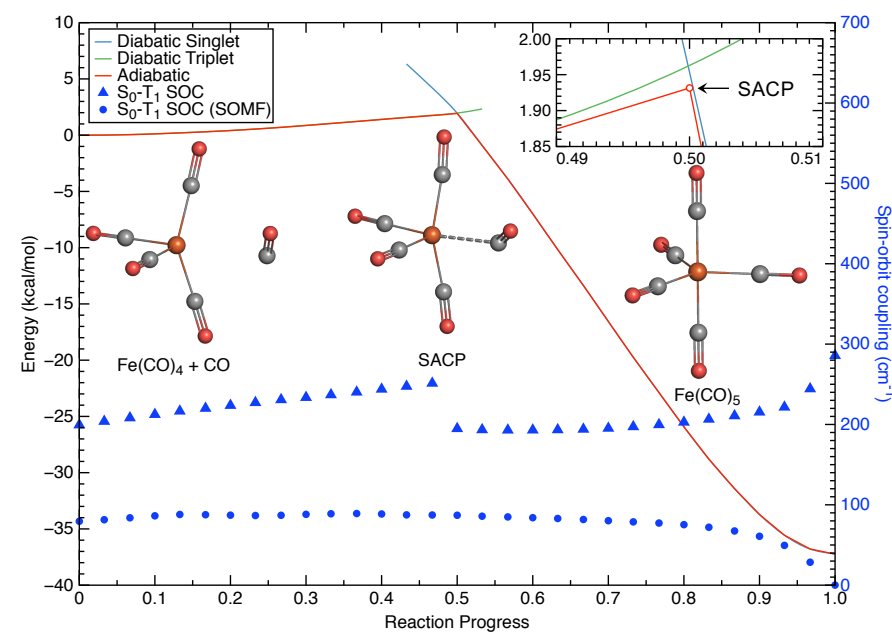

Figure 5. Energy profile and SOC strengths for the reaction process of $\mathrm{Fe}(\mathrm{CO})_{4}+\mathrm{CO} \rightarrow \mathrm{Fe}(\mathrm{CO})_{5}$ calculated at M06-L/def2-TZVPP level. Blue and green curves represent energies for diabatic singlet and triplet states, respectively. Red curve stands for the spinadiabatic state energy. Blue triangle dots show the change in the $\mathrm{S}_{0^{-}}$ $\mathrm{T}_{1}$ spin-orbit coupling strength along the reaction path. Blue round dots correspond to the spin-orbit coupling calculated with spin-orbit mean-field (SOMF) Hamiltonian from the ORCA package for a comparison.

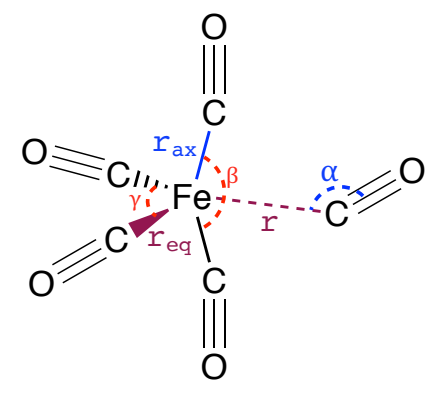

Figure 6. Schematic structure of the MECP/SACP geometry for the $\mathrm{Fe}(\mathrm{CO})_{5}$ spin-crossing reaction. Detailed geometric parameters are listed in Table II.

several other excitations beyond the $\mathrm{HOMO} \rightarrow$ LUMO transition.

\section{DISCUSSIONS}

As shown above, the restricted-and-shared-orbital formalism worked well to provide the spin-adiabatic states for three model spin-crossing reactions. It has a moderate computational cost, which amounts to two separate energy+force calculations (one singlet and one triplet). This method works the best when the low-spin and high-spin states (if solved separately) have very similar core and open-shell orbitals. Otherwise, a cusp would be found along the reaction energy profile.

\section{Why a cusp occurs on the reaction energy profile?}

In our model, the same set of spin-restricted molecular orbitals are employed to construct the low-spin and high-spin electronic states. It allows the energy of the spin-adiabatic state to be fully variational to molecular orbital coefficients and thus greatly simplifies the calculation of its analytical gradient. However, this would lead to a cusp on the spinadiabatic reaction energy profile, meaning that the gradient with respect to the reaction coordinate is discontinuous at the crossing point. So why does this happen?

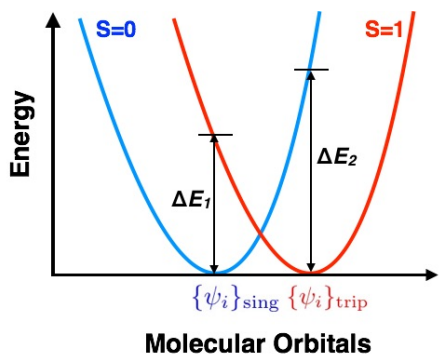

Figure 7. Schematic illustration of the spin-restricted closed-shell singlet and open-shell triplet energy surfaces varying with molecular orbitals at the spin-adiabatic cusp point.

In our spin-adiabatic state calculations, it is convenient to use converged SCF orbitals, from a previous closed-shell singlet calculation, $\left\{\Psi_{i}\right\}_{\text {sing }}$, or restricted open-shell triplet calculation, $\left\{\Psi_{i}\right\}_{\text {trip }}$, as the initial guess for the computation of spin-adiabatic states. Let us think about how these mixed-spin states get constructed with these initial orbitals at the spin-adiabatic cusp point (SACP). As illustrated in Figure 7 , the singlet orbitals, $\left\{\Psi_{i}\right\}_{\text {sing }}$, are only optimal for the singlet state, leaving the corresponding restricted open-shell triplet state (which is non-variational) substantially higher in energy. At this set of orbitals, the singlet-triplet energy gap $\Delta E_{1}$ (which is roughly the $S_{0} \longrightarrow T_{1}$ excitation energy within time-dependent density functional theory) ranges from 5.68 to $11.67 \mathrm{kcal} / \mathrm{mol}$ for three test systems (Table III). Meanwhile, the triplet orbitals, $\left\{\Psi_{i}\right\}_{\text {trip }}$, lead to a gap of $\Delta E_{2}$ (which is roughly the energy change from the triplet reference to the closed-shell singlet in spin-flip density functional theory calculations ${ }^{78-80}$ ) in the range of 6.0 to $11.93 \mathrm{kcal} / \mathrm{mol}$ for our test systems.

From Table III, it is clear that for both $\mathrm{N}_{2} \mathrm{O}$ and $\mathrm{CH}_{2}$ systems, such singlet-triplet energy gaps (between one variational state and another non-variational one) are 15-20 times larger than the spin-orbit coupling. For the $\mathrm{Fe}(\mathrm{CO})_{5}$, the gap is nearly 50 times larger than $\mathrm{V}^{\mathrm{SOC}}$. At this limit, the energy of the spin-adiabatic state in Eq. 3 becomes

$$
E^{\text {adia }}=\min \left(E^{\text {sing }}, E^{\text {trip }}\right)-\frac{\left(V^{\text {SOC }}\right)^{2}}{2(\Delta E)}
$$

The energy lowering from the spin-diabatic state to the spinadiabatic states is thus $-\frac{1}{2}\left(V^{\text {SOC }}\right)^{2} /(\Delta E)$, which is $30-100$ 
Table III. Singlet-triplet energy gap and Breit-Pauli one-electron spin-orbit coupling (both in $\mathrm{kcal} / \mathrm{mol}$ ) with converged SCF orbitals (illustrated in Figure 7) from closed-shell singlet and restricted openshell triplet calculations for three test systems. Spin-orbit coupling values are also shown in $\mathrm{cm}^{-1}$ in round brackets.

\begin{tabular}{lccccc}
\hline \hline \multirow{2}{*}{ Molecule } & \multicolumn{2}{c}{ Singlet } & & \multicolumn{2}{c}{ Triplet } \\
\cline { 2 - 3 } \cline { 5 - 6 } & $\left|\Delta E_{1}\right|$ & $\mathrm{V}^{\text {SOC }}$ & & $\left|\Delta E_{2}\right|$ & $V^{\text {SOC }}$ \\
\hline $\mathrm{N}_{2} \mathrm{O}$ & 8.84 & $0.55(192.3)$ & & 7.93 & $0.61(213.0)$ \\
$\mathrm{CH}_{2}$ & 5.68 & $0.12(43.5)$ & & 6.00 & $0.14(47.9)$ \\
$\mathrm{Fe}(\mathrm{CO})_{5}$ & 11.67 & $0.53(185.9)$ & & 11.93 & $0.66(230.1)$ \\
\hline \hline
\end{tabular}

times smaller than $V^{\text {SOC }}$ for the three systems. With an already rather small value for the spin-orbit coupling in the range of 0.12 to $0.66 \mathrm{kcal} / \mathrm{mol}$ for the three systems, this causes the spin-adiabatic surfaces to deviate less than 0.03 $\mathrm{kcal} / \mathrm{mol}$ from the spin-diabatic surfaces, which was clearly shown in Figures 3, 4, and 5.

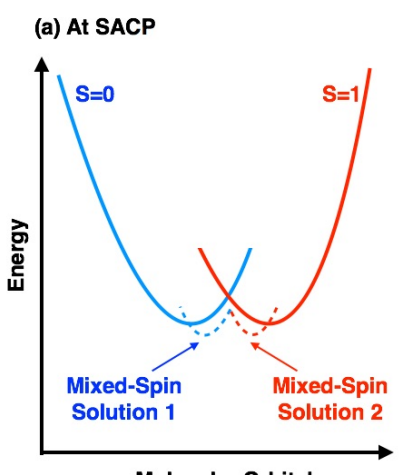

Molecular Orbitals

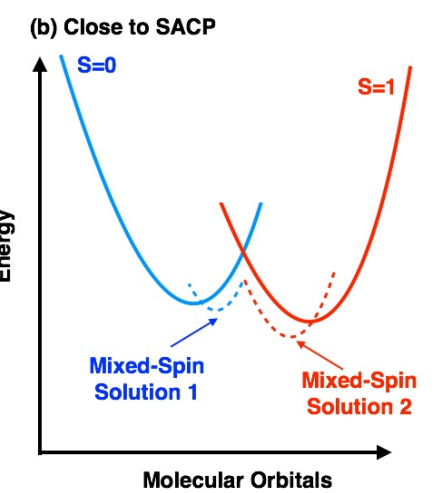

Molecular Orbitals
Figure 8. Schematic illustration of the spin-restricted closed-shell singlet and open-shell triplet, and the corresponding SCF solutions for the mixed-spin state at (or close to) the cusp. The spin-adiabatic energy surface corresponds to the lower-energy mixed-spin solution at each geometry.

With initial orbitals from either singlet or triplet calculations, the eigenvector of the 2-state SOC Hamiltonian in Eq. 2 is dominated by the lower-energy spin-diabatic state, with a mixing coefficient ratio of $\left[1-\left(V^{\text {SOC }}\right) /(2 \Delta E)\right]$ : $\left[\left(V^{\text {SOC }}\right) /(2 \Delta E)\right]$. Subsequent SCF optimizations (as described in Section IIC and shown schematically in Figure 8) only bring very small changes to the molecular orbitals. In the end, at each geometry at or close to the crossing point, there are two SCF solutions for the mixed-spin state: one close to the singlet spin-diabat and the other close to the triplet one. At the crossing point, the lower-energy spin-adiabatic state suddenly moves from one minimum to another. Therefore, a cusp occurs.

\section{How to obtain a more smooth reaction energy profile?}

As long as the low-spin and high-spin electronic states are required to share the same set of molecular orbitals, more sophisticated Breit-Pauli spin-orbitals models, like the SOMF model, will still produce a cusp around the crossing point. This is expected because, as shown in Figure 5, the SOMF SOC model produces even smaller SOC values than the unscaled Breit-Pauli one-electron model.

However, it is highly desirable to have a smooth potential surface, which allows us to use standard algorithms for locating the transition state and mapping the minimum energy pathway (as illustrated schematically in Figure 1b). The key to a smooth spin-adiabatic potential energy is to allow the two electronic states adopting different sets of molecular orbitals. Mathematically, this would allow diagonal elements in the 2state Hamiltonian (Eq. 2) to be equal at the crossing point, whereby the two states can be mixed evenly.

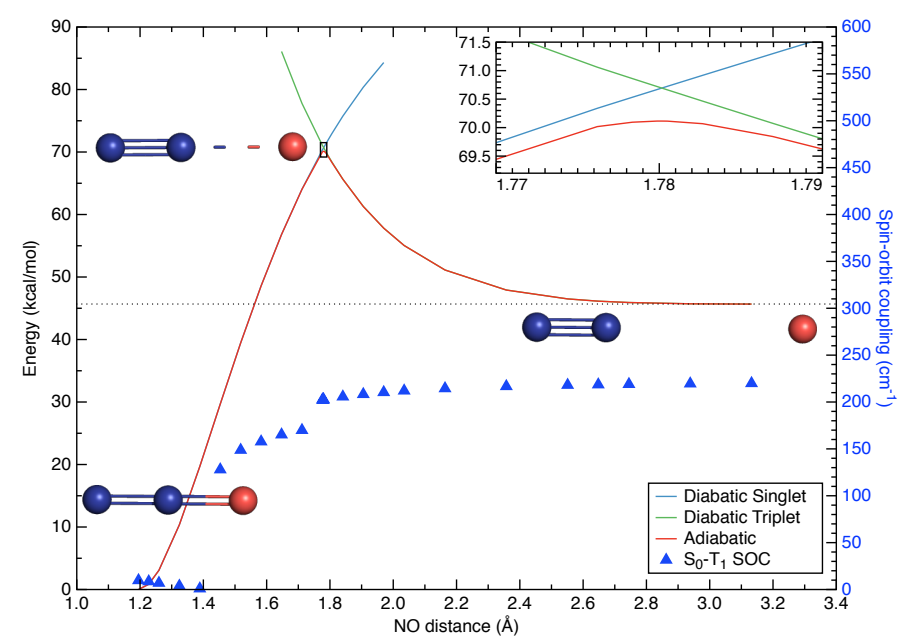

Figure 9. Energy profile and $\mathrm{SOC}$ strengths for $\mathrm{N}_{2} \mathrm{O}$ predissociation calculated at B3LYP/6-31+G(d) level. Red curve stands for the energy of the spin-adiabatic state from mixing separately-optimized spin-diabatic states.

When different sets of spin-restricted orbitals are used to describe the singlet and triplet states of a molecule, one can approximate the spin-orbit coupling as

$$
\begin{aligned}
V_{m}^{\mathrm{SOC}}= & {\left[\operatorname{Det}\left(\mathbf{S}\left({ }^{\mathrm{s}} c ;^{\mathrm{t}} c\right)\right)\right]^{2}\left[\left\langle\left.{ }^{\mathrm{s}} a\right|^{\mathrm{t}} b\right\rangle\left\langle{ }^{\mathrm{s}} \bar{a}\left|\hat{V}_{m}^{\mathrm{so}}\right|{ }^{\mathrm{t}} a\right\rangle\right.} \\
& \left.+\left\langle\left.{ }^{\mathrm{s}} a\right|^{\mathrm{t}} a\right\rangle\left\langle{ }^{\mathrm{s}} \bar{a}\left|\hat{V}_{m}^{\mathrm{so}}\right|{ }^{\mathrm{t}} b\right\rangle\right]
\end{aligned}
$$

where ${ }^{\mathrm{s}} a$ and ${ }^{\mathrm{s}} \bar{a}$ correspond to a doubly-occupied orbital from the singlet that becomes approximately singly occupied in the triplet. In other words, it spatially overlaps the most with ${ }^{\mathrm{t}} a$ and ${ }^{t} b$, two singly-occupied orbitals for the triplet. ${ }^{39}$ In the above equation, ${ }^{\mathrm{s}} c$ and ${ }^{\mathrm{t}} c$ stand for other doubly-occupied (core) orbitals for two spin states, $\operatorname{Det}\left(\mathbf{S}\left({ }^{\mathrm{s}} c ;{ }^{\mathrm{t}} c\right)\right)$, measures the overlap between the two core-orbital subspaces. Using such SOC values, one can obtain a more smooth reaction energy profile in Figure 9 for $\mathrm{N}_{2} \mathrm{O}$ predissociation. Figure $\mathrm{S} 1$ in the supporting information shows a similarly smooth energy profile for the $\mathrm{Fe}(\mathrm{CO})_{5}$ system. The analytical gradient of these 
models are being developed, and will be reported in a subsequent publication.

\section{CONCLUSIONS}

In this work, a new method is explored for the construction of spin-adiabatic states in the modeling of spin-crossing reactions. The main characteristics of this method are:

- the unscaled Breit-Pauli one-electron operator was used to evaluate the spin-orbit coupling between two spindiabatic states;

- the low-spin (e.g. closed-shell singlet) and high-spin (e.g. restricted open-shell triplet) states are defined using the same set of molecular orbitals;

- the energy of spin-adiabatic states within a resultant 2-state Hamiltonian is fully variational with respect to molecular orbitals, which enabled a simpler evaluation of the analytical energy gradient.

Using this method, the spin-adiabatic reaction pathway was readily obtained for three model spin-crossing systems: $\mathrm{N}_{2} \mathrm{O}, \mathrm{CH}_{2}$ and $\mathrm{Fe}(\mathrm{CO})_{5}$, where the unscaled Breit-Pauli oneelectron SOC value was found to vary noticeably along the pathway. Due to small Breit-Pauli coupling values, and more importantly, the large difference between the diagonal elements in the 2-state Hamiltonian (arising from the use of a single set of molecular orbitals in low-spin and high-spin diabatic states), the mixing between two spin-diabats is very weak. In all three systems, the spin-adiabatic state energy differs from the corresponding spin-diabatic states by no more than 0.03 $\mathrm{kcal} / \mathrm{mol}$. This causes a cusp at the crossing point, which can only be located using the MECP-type algorithm

In general, our work reinforces the attractiveness of building spin-adiabtic energy surfaces for studying spin-crossing reactions ${ }^{16,18-22}$ as well as the feasibility of computing spinorbit coupling on the fly within the Breit-Pauli one-electron operator framework. ${ }^{41,42}$ However, in order to build more smooth spin-adiabtic potential energy surfaces using the Breit-Pauli one-electron operator so that standard transition state search and reaction pathway optimization algorithms can be utilized, more general methods need to be developed. Specifically, these methods would involve different molecular orbitals for different spin-diabats and thus require the solution of $z$-vector equations in the analytical gradient evauations.

\section{ACKNOWLEDGEMENTS}

The computing for this project was performed at the OU Supercomputing Center for Education \& Research (OSCER). YS thanks Drs. Joe Subotnik and Kenneth Nicholas for helpful discussions.

\section{FUNDING INFORMATION}

YT and YS are supported by the National Institutes of Health under grant No. P20GM1036040. YS is also supported by the Oklahoma Center for the Advancement of Science and Technology under grant No. HR18-130, and the Office of the Vice President of Research and the College of Art and Sciences at the University of Oklahoma (OU). NB is supported by National Science Foundation under Grant No. CHE-1764365 and by the National Science Foundation Graduate Research Fellowship Program under Grant No. DGE1321851.

\section{REFERENCES}

${ }^{1}$ Z. Yang, B. Eichelberger, O. Martinez, M. Stepanovic, T. P. Snow, and V. M. Bierbaum, "The Influence of Spin Effects on the Gas Phase Reactions of Carbanions with N and O Atoms," J. Am. Chem. Soc. 132, 5812-5819 (2010).

${ }^{2}$ A. Hernández-Ortega, M. G. Quesne, S. Bui, D. J. Heyes, R. A. Steiner, N. S. Scrutton, and S. P. de Visser, "Catalytic Mechanism of Cofactor-Free Dioxygenases and How They Circumvent Spin-Forbidden Oxygenation of Their Substrates," J. Am. Chem. Soc. 137, 7474-7487 (2015).

${ }^{3}$ R. Poli and J. N. Harvey, "Spin Forbidden Chemical Reactions of Transition Metal Compounds. New Ideas and New Computational Challenges," Chem. Soc. Rev. 32, 1-8 (2003).

${ }^{4}$ N. Strickland and J. N. Harvey, "Spin-Forbidden Ligand Binding to the Ferrous-Heme Group: Ab Initio and DFT Studies,' J. Phys. Chem. B 111, 841-852 (2007).

${ }^{5}$ E. I. Solomon, T. C. Brunold, M. I. Davis, J. N. Kemsley, S.-K. Lee, N. Lehnert, F. Neese, A. J. Skulan, Y.-S. Yang, and J. Zhou, "Geometric and Electronic Structure/Function Correlations in Non-Heme Iron Enzymes," Chem. Rev. 100, 235-350 (2000).

${ }^{6}$ E. I. Solomon, D. E. Heppner, E. M. Johnston, J. W. Ginsbach, J. Cirera, M. Qayyum, M. T. Kieber-Emmons, C. H. Kjaergaard, R. G. Hadt, and L. Tian, "Copper Active Sites in Biology," Chem. Rev. 114, 3659-3853 (2014).

${ }^{7}$ T. Saito and W. Thiel, "Quantum Mechanics/Molecular Mechanics Study of Oxygen Binding in Hemocyanin," J. Phys. Chem. B 118, 5034-5043 (2014).

${ }^{8}$ P. Pokhilko, R. Shannon, D. Glowacki, H. Wang, and A. I. Krylov, "SpinForbidden Channels in Reactions of Unsaturated Hydrocarbons with $\mathrm{O}$ ( ${ }^{3}$ P)," J. Phys. Chem. A 123, 482-491 (2019).

${ }^{9}$ C. M. Marian, "Spin-orbit Coupling and Intersystem Crossing in Molecules: Spin-Orbit Coupling,” WIRES Comput. Mol. Sci. 2, 187-203 (2012).

${ }^{10}$ B. Mussard and S. Sharma, "One-Step Treatment of Spin-Orbit Coupling and Electron Correlation in Large Active Spaces," J. Chem. Theory Comput. 14, 154-165 (2018).

${ }^{11}$ N. Koga and K. Morokuma, "Determination of the Lowest Energy Point on the Crossing Seam Between Two Potential Surfaces Using the Energy Gradient," Chem. Phys. Lett. 119, 371-374 (1985).

${ }^{12}$ D. R. Yarkony, "Systematic Determination of Intersections of Potential Energy Surfaces Using a Lagrange Multiplier Constrained Procedure,” J. Phys. Chem. 97, 4407-4412 (1993).

${ }^{13}$ M. J. Bearpark, M. A. Robb, and H. Bernhard Schlegel, "A Direct Method for the Location of the Lowest Energy Point on a Potential Surface Crossing," Chem. Phys. Lett. 223, 269-274 (1994).

${ }^{14}$ J. N. Harvey, M. Aschi, H. Schwarz, and W. Koch, "The Singlet and Triplet States of Phenyl Cation. A Hybrid Approach for Locating Minimum Energy Crossing Points Between Non-interacting Potential Energy Surfaces," Theor. Chem. Acc. 99, 95-99 (1998).

${ }^{15}$ J. N. Harvey, "Spin-Forbidden Reactions: Computational Insight into Mechanisms and Kinetics," WIREs Comput. Mol. Sci. 4, 1-14 (2014).

${ }^{16}$ C. A. Gaggioli, L. Belpassi, F. Tarantelli, D. Zuccaccia, J. N. Harvey, and P. Belanzoni, "Dioxygen Insertion into the Gold(I)-hydride Bond: Spin Or- 
bit Coupling Effects in the Spotlight for Oxidative Addition," Chem. Sci. 7, 7034-7039 (2016).

${ }^{17}$ B. O. Roos and P.- Malmqvist, "On the Effects of Spin-Orbit Coupling on Molecular Properties: Dipole Moment and Polarizability of $\mathrm{PbO}$ and Spectroscopic Constants for the Ground and Excited States," in Adv. Quantum Chem., Vol. 47 (Elsevier, 2004) pp. 37-49.

${ }^{18}$ C. A. Gaggioli, L. Belpassi, F. Tarantelli, J. N. Harvey, and P. Belanzoni, "The Ligand Effect on the Oxidative Addition of Dioxygen to Gold(I)hydride Complexes," Dalton Trans. 46, 11679-11690 (2017).

${ }^{19}$ C. A. Gaggioli, L. Belpassi, F. Tarantelli, J. N. Harvey, and P. Belanzoni, "Spin-Forbidden Reactions: Adiabatic Transition States Using Spin-Orbit Coupled Density Functional Theory,” Chem. Eur. J. 24, 5006-5015 (2018).

${ }^{20}$ B. Yang, L. Gagliardi, and D. G. Truhlar, "Transition States of SpinForbidden Reactions," Phys. Chem. Chem. Phys. 20, 4129-4136 (2018).

${ }^{21}$ T. Takayanagi and T. Nakatomi, "Automated Reaction Path Searches for Spin-forbidden Reactions: Automated Reaction Path Searches," J. Comput. Chem. 39, 1319-1326 (2018).

${ }^{22}$ T. Nakatomi, S. Koido, Y. Watabe, and T. Takayanagi, "Spin-Inversion Mechanisms in the Reactions of Transition Metal /Cations ( $\mathrm{Sc}+, \mathrm{Ti}+, \mathrm{V}+$, $\mathrm{Cr}+, \mathrm{Mn}+, \mathrm{Fe}+, \mathrm{Co}+, \mathrm{Ni}+$, and $\mathrm{Cu}+$ ) with OCS in the Gas Phase: A Perspective from Automated Reaction Path Search Calculations," Int. J. Quantum Chem. 119, e25908 (2019).

${ }^{23}$ J. Baker, "An Algorithm for the Location of Transition States," J. Comput. Chem. 7, 385-395 (1986).

${ }^{24}$ C. Peng and H. Bernhard Schlegel, "Combining Synchronous Transit and Quasi-Newton Methods to Find Transition States," Israel J. Chem. 33, 449454 (1993).

${ }^{25}$ G. Henkelman and H. Jonsson, "A Dimer Method for Finding Saddle Points on High Dimensional Potential Surfaces Using Only First Derivatives," J. Chem. Phys. 111, 7010-7022 (1999).

${ }^{26}$ A. Heyden, A. T. Bell, and F. J. Keil, "Efficient Methods for Finding Transition States in Chemical Reactions: Comparison of Improved Dimer Method and Partitioned Rational Function Optimization Method," J. Chem. Phys. 123, 224101 (2005).

${ }^{27}$ J. Kastner and P. Sherwood, "Superlinearly Converging Dimer Method for Transition State Search," J. Chem. Phys. 128, 014106 (2008).

${ }^{28}$ B. Peters, A. Heyden, A. T. Bell, and A. Chakraborty, "A Growing String Method for Determining Transition States: Comparison to the Nudged Elastic Band and String Methods," J. Chem. Phys. 120, 7877-7886 (2004).

${ }^{29}$ A. Behn, P. M. Zimmerman, A. T. Bell, and M. Head-Gordon, "Incorporating Linear Synchronous Transit Interpolation into the Growing String Method: Algorithm and Applications," J. Chem. Theory Comput. 7, 40194025 (2011).

${ }^{30}$ P. M. Zimmerman, "Growing String Method with Interpolation and Optimization in Internal Coordinates: Method and Examples," J. Chem. Phys. 138, 184102 (2013).

${ }^{31}$ A. Behn, P. M. Zimmerman, A. T. Bell, and M. Head-Gordon, "Efficient Exploration of Reaction Paths via a Freezing String Method," J. Chem. Phys. 135, 224108 (2011).

${ }^{32}$ S. Mallikarjun Sharada, P. M. Zimmerman, A. T. Bell, and M. HeadGordon, "Automated Transition State Searches without Evaluating the Hessian," J. Chem. Theory Comput. 8, 5166-5174 (2012).

${ }^{33}$ E. van Lenthe, J. G. Snijders, and E. J. Baerends, "The Zero-Order Regular Approximation for Relativistic Effects: The Effect of Spin-Orbit Coupling in Closed Shell Molecules," J. Chem. Phys. 105, 6505-6516 (1996).

${ }^{34}$ M. Filatov and D. Cremer, "On the Physical Meaning of the ZORA Hamiltonian," Mol. Phys. 101, 2295-2302 (2003).

${ }^{35} \mathrm{P}$. Abegg, "Ab initio Calculation of Spin-Orbit Coupling Constants for Gaussian Lobe and Gaussian-Type Wave Functions," Mol. Phys. 30, 579596 (1975).

${ }^{36}$ T. R. Furlani and H. F. King, "Theory of Spin-Orbit Coupling. Application to Singlet-Triplet Interaction in the Trimethylene Biradical,' J. Chem. Phys. 82, 5577-5583 (1985).

${ }^{37}$ S. Koseki, M. W. Schmidt, and M. S. Gordon, "MCSCF/6-31g(d,p) Calculations of One-Electron Spin-Orbit Coupling Constants in Diatomic Molecules," J. Phys. Chem. 96, 10768-10772 (1992).

${ }^{38}$ D. G. Fedorov and M. S. Gordon, "A Study of the Relative Importance of One and Two-Electron Contributions to Spin-Orbit Coupling," J. Chem. Phys. 112, 5611-5623 (2000).
${ }^{39}$ S. Chiodo and N. Russo, "Determination of Spin-Orbit Coupling Contributions in the Framework of Density Functional Theory," J. Comput. Chem. 29, 912-920 (2008).

${ }^{40}$ X. Gao, S. Bai, D. Fazzi, T. Niehaus, M. Barbatti, and W. Thiel, "Evaluation of Spin-Orbit Couplings with Linear-Response Time-Dependent Density Functional Methods," J. Chem. Theory Comput. 13, 515-524 (2017).

${ }^{41}$ N. Bellonzi, G. R. Medders, E. Epifanovsky, and J. E. Subotnik, "Configuration Interaction Singles with Spin-Orbit Coupling: Constructing SpinAdiabatic States and Their Analytical Nuclear Gradients," J. Chem. Phys. 150, 014106 (2019).

${ }^{42}$ N. Bellonzi, S. Fatehi, Y. Shao, and J. E. Subotnik, "TD-DFT SpinAdiabats with Analytic Nonadiabatic Derivative Coupling," Under preparation.

${ }^{43}$ F. Neese, "Efficient and Accurate Approximations to the Molecular SpinOrbit Coupling Operator and Their Use in Molecular g-Tensor Calculations," J. Chem. Phys. 122, 034107 (2005).

${ }^{44}$ T. Kowalczyk, T. Tsuchimochi, P.-T. Chen, L. Top, and T. Van Voorhis, "Excitation Energies and Stokes Shifts from a Restricted Open-Shell KohnSham Approach," J. Chem. Phys. 138, 164101 (2013).

${ }^{45}$ D. Hait, T. Zhu, D. P. McMahon, and T. Van Voorhis, "Prediction of Excited-State Energies and Singlet-Triplet Gaps of Charge-Transfer States Using a Restricted Open-Shell Kohn-Sham Approach,” J. Chem. Theory Comput. 12, 3353-3359 (2016).

${ }^{46} \mathrm{M}$. Filatov and S. Shaik, "A Spin-Restricted Ensemble-Referenced Kohn-Sham Method and Its Application to Diradicaloid Situations," Chem. Phys. Lett. 304, 429-437 (1999).

${ }^{47}$ B. N. Plakhutin, E. V. Gorelik, and N. N. Breslavskaya, "Koopmans' Theorem in the ROHF Method: Canonical Form for the Hartree-Fock Hamiltonian," J. Chem. Phys. 125, 204110 (2006).

${ }^{48}$ T. Tsuchimochi and G. E. Scuseria, "ROHF Theory Made Simple," J. Chem. Phys. 133, 141102 (2010).

${ }^{49}$ J. Binkley, J. Pople, and P. Dobosh, "The Calculation of Spin-Restricted Single-Determinant Wavefunctions,” Mol. Phys. 28, 1423-1429 (1974).

${ }^{50}$ Y. Shao, Z. Gan, E. Epifanovsky, A. T. Gilbert, M. Wormit, J. Kussmann, A. W. Lange, A. Behn, J. Deng, X. Feng, D. Ghosh, M. Goldey, P. R. Horn, L. D. Jacobson, I. Kaliman, R. Z. Khaliullin, T. Kuś, A. Landau, J. Liu, E. I. Proynov, Y. M. Rhee, R. M. Richard, M. A. Rohrdanz, R. P. Steele, E. J. Sundstrom, H. L. Woodcock, P. M. Zimmerman, D. Zuev, B. Albrecht, E. Alguire, B. Austin, G. J. O. Beran, Y. A. Bernard, E. Berquist, K. Brandhorst, K. B. Bravaya, S. T. Brown, D. Casanova, C.-M. Chang, Y. Chen, S. H. Chien, K. D. Closser, D. L. Crittenden, M. Diedenhofen, R. A. DiStasio, H. Do, A. D. Dutoi, R. G. Edgar, S. Fatehi, L. Fusti-Molnar, A. Ghysels, A. Golubeva-Zadorozhnaya, J. Gomes, M. W. Hanson-Heine, P. H. Harbach, A. W. Hauser, E. G. Hohenstein, Z. C. Holden, T.-C. Jagau, H. Ji, B. Kaduk, K. Khistyaev, J. Kim, J. Kim, R. A. King, P. Klunzinger, D. Kosenkov, T. Kowalczyk, C. M. Krauter, K. U. Lao, A. D. Laurent, K. V. Lawler, S. V. Levchenko, C. Y. Lin, F. Liu, E. Livshits, R. C. Lochan, A. Luenser, P. Manohar, S. F. Manzer, S.-P. Mao, N. Mardirossian, A. V. Marenich, S. A. Maurer, N. J. Mayhall, E. Neuscamman, C. M. Oana, R. Olivares-Amaya, D. P. O'Neill, J. A. Parkhill, T. M. Perrine, R. Peverati, A. Prociuk, D. R. Rehn, E. Rosta, N. J. Russ, S. M. Sharada, S. Sharma, D. W. Small, A. Sodt, T. Stein, D. Stück, Y.-C. Su, A. J. Thom, T. Tsuchimochi, V. Vanovschi, L. Vogt, O. Vydrov, T. Wang, M. A. Watson, J. Wenzel, A. White, C. F. Williams, J. Yang, S. Yeganeh, S. R. Yost, Z.-Q. You, I. Y. Zhang, X. Zhang, Y. Zhao, B. R. Brooks, G. K. Chan, D. M. Chipman, C. J. Cramer, W. A. Goddard, M. S. Gordon, W. J. Hehre, A. Klamt, H. F. Schaefer, M. W. Schmidt, C. D. Sherrill, D. G. Truhlar, A. Warshel, X. Xu, A. Aspuru-Guzik, R. Baer, A. T. Bell, N. A. Besley, J.-D. Chai, A. Dreuw, B. D. Dunietz, T. R. Furlani, S. R. Gwaltney, C.-P. Hsu, Y. Jung, J. Kong, D. S. Lambrecht, W. Liang, C. Ochsenfeld, V. A. Rassolov, L. V. Slipchenko, J. E. Subotnik, T. Van Voorhis, J. M. Herbert, A. I. Krylov, P. M. Gill, and M. Head-Gordon, "Advances in Molecular Quantum Chemistry Contained in the Q-Chem 4 Program Package," Mol. Phys. 113, 184215 (2015).

${ }^{51}$ P. Pulay, "Convergence Acceleration of Iterative Sequences. The Case of SCF Iteration," Chem. Phys. Lett. 73, 393-398 (1980).

${ }^{52}$ T. Van Voorhis and M. Head-Gordon, "A Geometric Approach to Direct Minimization,” Mol. Phys. 100, 1713-1721 (2002).

${ }^{53}$ A. D. Becke, "Density-functional Exchange-Energy Approximation with Correct Asymptotic Behavior," Phys. Rev. A 38, 3098-3100 (1988). 
${ }^{54}$ A. D. Becke, "A New Mixing of Hartree-Fock and Local DensityFunctional Theories,” J. Chem. Phys. 98, 1372-1377 (1993).

${ }^{55}$ C. Lee, W. Yang, and R. G. Parr, "Development of the Colle-Salvetti Correlation-Energy Formula into a Functional of the Electron Density," Phys. Rev. B 37, 785-789 (1988).

${ }^{56}$ M. M. Francl, W. J. Pietro, W. J. Hehre, J. S. Binkley, M. S. Gordon, D. J. DeFrees, and J. A. Pople, "Self-Consistent Molecular Orbital Methods. XXIII. A Polarization-Type Basis Set for Second-Row Elements," J. Chem. Phys. 77, 3654-3665 (1982).

${ }^{57}$ R. Krishnan, J. S. Binkley, R. Seeger, and J. A. Pople, "Self-Consistent Molecular Orbital Methods. XX. A Basis Set for Correlated Wave Functions," J. Chem. Phys. 72, 650-654 (1980).

${ }^{58}$ Y. Zhao and D. G. Truhlar, "A New Local Density Functional for Maingroup Thermochemistry, Transition Metal Bonding, Thermochemical Kinetics, and Noncovalent Interactions," J. Chem. Phys. 125, 194101 (2006).

${ }^{59}$ F. Weigend and R. Ahlrichs, "Balanced Basis Sets of Split Valence, Triple Zeta Valence and Quadruple Zeta Valence Quality for H to Rn: Design and Assessment of Accuracy," Phys. Chem. Chem. Phys. 7, 3297 (2005).

${ }^{60} \mathrm{~F}$. Weigend, "Accurate Coulomb-fitting Basis Sets for $\mathrm{H}$ to Rn," Phys. Chem. Chem. Phys. 8, 1057 (2006).

${ }^{61}$ V. Lebedev, “Quadratures on a Sphere," USSR Comput. Math. Math. Phys. 16, 10-24 (1976).

${ }^{62}$ F. Neese, "The ORCA Program System, version 4.0," WIREs Comput. Mol. Sci. 8, e1327 (2018).

${ }^{63}$ H. W. Erving, "The Discoverer of Anaesthesia: Dr. Horace Wells of Hartford.” Yale J. Biol. Med. 5, 421-430 (1933).

${ }^{64}$ E. R. Fisher and E. Bauer, "On the Quenching of $\mathrm{O}\left({ }^{1} \mathrm{D}\right)$ by $\mathrm{N}_{2}$ and Related Reactions,” J. Chem. Phys. 57, 1966-1974 (1972).

${ }^{65}$ J. B. Delos, "On the Reactions of $\mathrm{N}_{2}$ with O," J. Chem. Phys. 59, 23652369 (1973).

${ }^{66}$ L. K. Cohen and H. O. Pritchard, "Comparison of Unimolecular Rates Calculated Classically and Quantally from the Same Potential Surface," J. Phys. Chem. 90, 990-992 (1986).

${ }^{67}$ A. H. H. Chang and D. R. Yarkony, "On the Electronic Structure Aspects of Spin-Forbidden Processes in $\mathrm{N}_{2}$ O,” J. Chem. Phys. 99, 6824-6831 (1993).

${ }^{68}$ D.-Y. Hwang and A. M. Mebel, "Ab Initio Study of the Reaction Mechanism of Singlet and Triplet $\mathrm{N}_{2} \mathrm{O}$ and Their Intersystem Crossing," Chem. Phys. 259, 89-97 (2000).
${ }^{69}$ A. J. Marks and D. L. Thompson, “A Trajectory Surface-Hopping Study of Mode Specificity in the Predissociation of $\mathrm{N}_{2} \mathrm{O}$,' J. Chem. Phys. 95, 80568064 (1991)

${ }^{70}$ H. S. Johnston, "Interpretation of the Data on the Thermal Decomposition of Nitrous Oxide,” J. Chem. Phys. 19, 663-667 (1951).

${ }^{71}$ D. Bourissou, O. Guerret, F. P. Gabbaï, and G. Bertrand, "Stable Carbenes," Chem. Rev. 100, 39-92 (2000).

${ }^{72}$ L. V. Slipchenko and A. I. Krylov, "Singlet-Triplet Gaps in Diradicals by the Spin-Flip Approach: A Benchmark Study,” J. Chem. Phys. 117, 4694 4708 (2002)

${ }^{73}$ Z. Havlas, J. W. Downing, and J. Michl, "Spin-Orbit Coupling in Biradicals. 2.1 Ab Initio Methodology and Application to 1,1-Biradicals: Carbene and Silylene,' J. Phys. Chem. A 102, 5681-5692 (1998).

${ }^{74}$ A. R. W. McKellar, P. R. Bunker, T. J. Sears, K. M. Evenson, R. J. Saykally, and S. R. Langhoff, "Far Infrared Laser Magnetic Resonance of Singlet Methylene: Singlet-Triplet Perturbations, Singlet-Triplet Transitions, and the Singlet-Triplet Splitting," J. Chem. Phys. 79, 5251-5264 (1983).

${ }^{75}$ T. A. Seder, A. J. Ouderkirk, and E. Weitz, "The Wavelength Dependence of Excimer Laser Photolysis of $\mathrm{Fe}(\mathrm{CO})_{5}$ in the Gas Phase. Transient Infrared Spectroscopy and Kinetics of the $\mathrm{Fe}(\mathrm{CO})_{x}(\mathrm{x}=4,3,2)$ Photofragments," J. Chem. Phys. 85, 1977-1986 (1986).

${ }^{76}$ J. N. Harvey and M. Aschi, "Modelling Spin-Forbidden Reactions: Recombination of Carbon Monoxide with Iron Tetracarbonyl,' Faraday Discuss. 124, 129-143 (2003).

${ }^{77}$ J. N. Harvey, "Spin-Forbidden Reactions in Transition Metal Chemistry," in Computational Organometallic Chemistry, edited by T. R. Cundari (Marcel Dekker, Inc., New York, 2001) Chap. 12, pp. 291-321.

${ }^{78}$ Y. Shao, M. Head-Gordon, and A. I. Krylov, "The Spin-Flip Approach within Time-Dependent Density Functional Theory: Theory and Applications to Diradicals," J. Chem. Phys. 118, 4807-4818 (2003).

${ }^{79}$ F. Wang and T. Ziegler, "Time-Dependent Density Functional Theory Based on a Noncollinear Formulation of the Exchange-Correlation Potential," J. Chem. Phys. 121, 12191 (2004).

${ }^{80}$ Y. A. Bernard, Y. Shao, and A. I. Krylov, "General Formulation of SpinFlip Time-Dependent Density Functional Theory Using Non-Collinear Kernels: Theory, Implementation, and Benchmarks," J. Chem. Phys. 136, 204103 (2012). 